\title{
Burn-out im Arztberuf
}

\section{Mirjam Tanner}

Dr. med., Fachärztin für Psychiatrie und Psychotherapie, Leitungsausschuss ReMed

Ein Kaderarzt, der die Orthopädische Abteilung an einem Spital leitet, darf aufgrund der Corona-Krise keine Operationen mehr durchführen. Er beginnt an sich und seinen Fähigkeiten als Arzt zu zweifeln, vermutet ein «Burn-out» - und findet Hilfe in der Beratung durch ReMed.

In der Kontaktaufnahme mit ReMed per E-Mail hiess es, abgesehen von den notwendigen Kontaktangaben, nur trocken: «Burn-out». Mit der Erfahrung im Hintergrund, dass einem Burn-out in der Regel eine lange, konflikthafte und bedeutsame Geschichte zugrunde liegt, nahm ich interessiert Kontakt mit dem Kollegen auf. Dieser legte die anfängliche Zurückhaltung rasch $\mathrm{ab}$ und wollte das Angebot der zweistündigen Beratung durch ReMed am liebsten im direkten Kontakt in meiner Praxis wahrnehmen.

\section{Ethischer Anspruch und Vulnerabilität}

Als Kaderarzt leitet der Kollege die Orthopädische Abteilung an einem Spital. Die hohe Verantwortung im Arztberuf bedeutete ihm schon immer sehr viel, motivierte ihn für den Arztberuf und fordert ihn auf erfül-

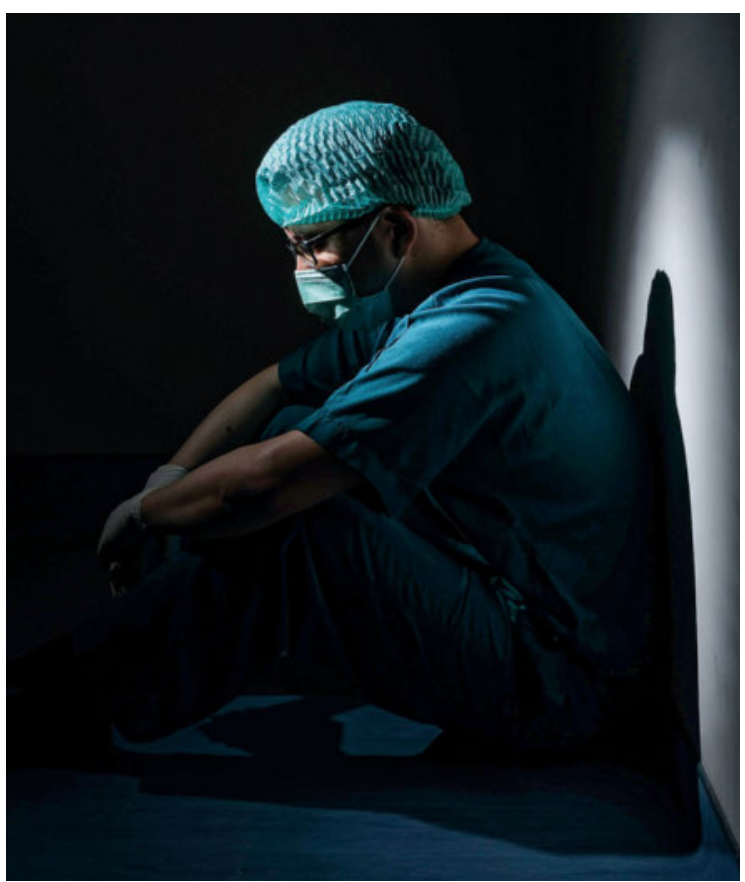

In der Krise finden Ärzte Unterstützung bei ReMed. lende Weise nach wie vor heraus. "Ich würde mich schon als Mensch mit extrem hohen ethischen und moralischen Ansprüchen an sich selbst - und auch an andere - beschreiben. Sprechen Sie mal mit meiner Frau oder unseren Kindern - die können Ihnen ein Lied davon singen», lacht er etwas verlegen. «Im Beruf war es für mich so zum Beispiel nie schwierig und sogar eine Selbstverständlichkeit, fachliche Grenzen bei mir sowie bei Kolleginnen und Kollegen offen und ehrlich zu erkennen und anzusprechen. Die «Doktor-Alleswisser-Fassade> zieht bei mir gar nicht. In meinem Team unterstützen und stärken wir uns gegenseitig. Eine erstklassige und an den Bedürfnissen der Patientinnen und Patienten orientierte Versorgung sowie die höchstmögliche Sicherheit waren und bleiben zwei meiner höchsten Werte als Chirurg. Das hat wohl im Verlauf meiner Karriere zu einer eher restriktiven Operationsindikationsstellung geführt. So habe ich es zumindest lange gesehen und das haben mir auch meine Kolleginnen und Kollegen entsprechend gespiegelt.

\section{Neue, unangenehme Erkenntnisse}

Jetzt nach eineinhalb Jahren Corona kämpfe ich mit völlig neuartigen, heftigen Selbstzweifeln. Und ich komme plötzlich mit meiner Arbeit und dem Druck, unter dem ich mich gegenwärtig wiederfinde, nicht mehr zurecht. Ich erwache in der Nacht schweissgebadet und grüble stundenlang unergiebig, vor allem über mögliche zweifelhafte Behandlungsempfehlungen in der Vergangenheit. Zum Beispiel die 57-jährige Patientin, mit der eine Knieprothesenoperation vereinbart wurde. Im Zuge der Coronaeinschränkungen auf notfallmässige Eingriffe hatte sie ihre Knieprobleme offenbar durch konservative Behandlung so weit in den Griff bekommen, dass sie auch später, als wir wieder operieren durften, auf die Operation verzichtete. Ich war aber damals überzeugt, dass sie bei ihrer Situation die Operation benötigt. 
Ich fühle mich elend, zweifle an mir selbst und finde keinen Ausweg mehr aus einer vernichtenden Abwärtsspirale. Ein guter Kollege von der Anästhesie, von dem ich es überhaupt nicht erwartet hätte, verriet mir, dass er wunderbar schlafe mit einer Mischung aus Scotch und Lexotanil vor dem Zubettgehen.»

Der Kollege liess keine Zweifel daran, dass eine solche Selbstmedikation für ihn keine Option ist, um seinen Schlafproblemen zu begegnen und dass sie dem eigenen Anspruch, den er an sich selber stellt, voll und ganz widerspricht. In der zweiten Stunde konnten wir die Hintergründe für das «Burn-out» weiter klären und immer besser verstehen.

\section{Die Zwickmühle}

Die Coronakrise mit der auferlegten Phase des Operationsstopps für Wahleingriffe wurde zum Ausgangspunkt einer tiefen moralischen Sinnkrise. Gerade er, der seinen Patientinnen und Patienten doch sehr zurückhaltend Operationen empfahl, musste zweierlei höchst schwerverdauliche Folgen aus dem Operationsstopp erleben. Die erste, eher etwas weniger zermürbende, war mitzubekommen, wie sich einige Patientinnen und Patienten im Zusammenhang mit dem Operationsstopp von ihm abwendeten und die Behandlungen anderweitig weiterführten. Er war enttäuscht von deren Opportunismus und darüber, wie wenig sie sein Engagement würdigten. Selbstkritisch fragte er sich, ob er rückblickend Patientinnen und $\mathrm{Pa}$ tienten Eingriffe nahegelegt und zugemutet hatte, die mit allergrösster Wahrscheinlichkeit unnötig gewesen wären. Diese deprimierende Erkenntnis wird noch erdrückender durch den aktuellen zunehmenden ökonomischen Druck, der auf ihm lastet - dieser ist inzwischen so gross wie noch nie. Dieser Indikationsstress

CH-3000 Bern 15 Tel. 0313591200 info[at]swiss-remed.ch

\section{ReMed-Intervisionen für Erstberatende und Netzwerkmitglieder}

Neben den Unterstützungsangeboten für ratsuchende Ärztinnen und Ärzte führt ReMed auch regionale Intervisionen zum Erfahrungsaustausch für Kolleginnen und Kollegen durch, die Ärztinnen und Ärzte als Patienten betreuen. Diese ermöglichen Vernetzung und Bildung von Peer-Groups (jeweils 6-8Teilnehmer, 2-3 Treffen pro Jahr), welche gemeinsam Fallfragen zu Mentoring, Coaching, Beratung, Therapie oder anderen Aspekten (juristisch, versicherungsrechtlich etc.) erarbeiten. Setzen Sie sich mit uns in Verbindung, nehmen Sie an einer Sitzung teil und lernen Sie unsere Arbeit kennen. Kontakt und Anmeldung: info[at]swissremed.ch. Mögliche nächste Daten 2022:

Donnerstag, 10. März 2022

Donnerstag, 21. April 2022

Donnerstag, 5. Mai 2022

Donnerstag, 9. Juni 2022

Donnerstag, 15. September 2022

Donnerstag, 10. November 2022

14-18 Uhr im Hotel Central Plaza in Zürich

verunsichert ihn und löst Existenzängste aus. Das Gefühl, nicht mehr primär Patientinnen und Patienten zu behandeln, sondern der Spitalleitung, mehrheitlich mit Nicht-Medizinern besetzt, Fallzahlen und eine vorgegebene Anzahl Operationen liefern zu müssen, steht in krassem Widerspruch zu seinen persönlichen beruflichen Wertvorstellungen.

\section{Mögliche emotionelle Konflikte}

Dieser Kollege mit guter Introspektionsfähigkeit erkannte den emotionellen Konflikt hinter seinem «Burn-out» sehr schnell. Gegenwärtig geht er diesem in einem von ReMed vermittelten Coaching weiter nach. Andere solche Konflikte, die uns bei ReMed als "Burn-out» begegnen, sind beispielsweise heruntergespielte, verleugnete oder schlecht wahrgenommene berufsbezogene Ängste, Enttäuschung und Sinnkrisen im Zusammenhang mit dem Arztberuf, Überforderung bei der Karriereplanung sowie grosse soziale Isolation und Einsamkeit.

Bildnachweis

Mulyadi / Unsplash 